\title{
Spontaneous Pnemoperitoneum after Blunt Trauma Chest - Diagnostic Dilemma-Role of Diagnostic Laparoscopy - Case Report
}

\author{
Samir R Nayak*, Mishra Anindita, Dilip Kumar Soren and Babu Nagendra S
}

Department of general surgery, GSL medical college and hospital, NH-5, Lakshmipuram,Rajahmundry, India

\begin{abstract}
Introduction: Pneumoperitoneum is a striking feature of hollow viscous perforation and may need of immediate surgical intervention. Blunt trauma chest with pneumoperitoneum without evidence of hollow viscous perforation is unusual and the condition is called spotaneous pneumoperitoneum.

Case Presentation: A 29 year male presented to the emergency department after a road traffic accident with hypotension and respiratory distress. Clinically there was surgical emphysema associated with diminished breath sound over left half of the chest and multiple contusions over left hypochondrium and left flank. Bedside X- ray showed fracture of ribs on left side with pneumothorax and air under both the dome of diaphragm. Emergency tube thoracostomy done and respiratory symptoms improved. Further patient evaluated with Ultrasound abdomen and Computerized tomogram of abdomen. Dilated bowels, gross pneumoperitoneum with minimal fluid collection was noted. Diagnostic laparoscopy done to find out hollow viscous perforation or diaphragm injury but to the surprising hollow viscous and diaphragm found to be normal.
\end{abstract}

Case Discussion: Pneumothorax and pnemoperitoneum with presence of abdominal contusions make the surgeon in dilemma for choosing conservative or therapeutic approach.

Conclusion: Diagnostic laparoscopy with systemic exploration of abdominal organs and spaces will help in diagnosis and mandatory laparotomy may avoided.

Keywords: Pneumoperitoneum; Blunt trauma chest; Diagnostic laparoscopy

\section{Introduction}

Pneumoperitoneum(pp) denotes an abnormal collection of air in the peritoneal cavity. It results s from a perforated hollow viscous in $90 \%$ of the cases and requires immediate surgical intervention [1] Spontaneous/idiopathic pneumoperitoneum associated with pneumothorax secondary to blunt trauma is rare. In this case the thoracic air dissects retroperitonealy or leaks directly through diaphragm. The diagnosis of Spontaneous pnemoperitoneum (SP) is usually made after negative laparotomy results.SP with a signs of peritonitis makes a therapeutic dilemma between conservative or non-conservative treatment. Doing a diagnostic laparoscope in this situation is safety and a major laparotomy may be avoided.

\section{Case Presentation}

A 29-year male presented to emergency department after a collision with lorry. He was in causality with respiratory distress, multiple abrasions over left half of chest and abdomen. His coma scale was $15 / 15$, pulse rate was 120 per minute regular, and Blood pressure was $90 \mathrm{~mm} \mathrm{Hg}$ systolic, respiratory rate was $40 /$ minute and oxygen saturation was70 \% with 5 liters of oxygen. Chest auscultation showed crepitus on left side with diminished breath sound at left infrascapular and midaxillry region. On examination of abdomen, there were imprint abrasions of tyre marks over the left hypochondrium and flank. Diffuse abdominal tenderness on palpation with obliteration of liver dullness. Bowel sound was sluggish. Pelvic compression test was negative. Spine examination appears normal. Resuscitation started to correct the hypotension. Bedside X-ray chest showed fracture of $7^{\text {th }} 8^{\text {th }} 9^{\text {th }}$ rib on left side with pneumothorax. He underwent placement of Inter Costal Tube Drainage (ICTD) on left side which promptly improved respiratory symptoms. After fluid resuscitation and intercostal tube placement, pulse rate was $100 /$ minute, blood pressure improved to
100/70 mm Hg.Oxygen saturation increased up to $98 \%$. Reevaluation of abdomen reveled tenderness all over abdomen with obliteration of liver dullness. Bedside echo excludes the pericardial effusions. X ray abdomen image demonstrated gas under both the dome of diaphragm. Ultrasound abdomen and computerized tomography scan showed gross pnemoperitoneum, minimal fluid in right sub diaphragmatic region.

Polytrauma patient with tyre marks over the abdomen and pneumoperitoneum prompted us to do the diagnostic laparoscopy. Diagnostic laparoscopy done with the $10 \mathrm{~mm}$ scope infraumblically. Liver and spleen found to be normal. Blood tinged fluid at right sub diaphragmatic region, no intestinal contents. The stomach and duodenum were fully mobilized, and the lesser sac explored. No perforation found in the distal esophagus, stomach or duodenum. The small and large bowel examined, but no leakage was observed. Diaphragm searched but no rent/ tears detected. Hence, the therapeutic procedure abandoned. All the laparoscopic findings were video recorded for review. Patient gradually improved. The postoperative course was uneventful, and the patient showed a significant and prompt recovery. Next postoperative day spirometry exercise started and patient kept on liquids. Repeat chest and abdominal X-rays

*Corresponding author: Samir Ranjan Nayak, Dept of General surgery, GSL Medical College, NH-5, Lakshmipuram, Rajahmundry, EG dist, 533294, Andhra Pradesh, India, Tel: 919550521218; E-mail: drsamirnayak@gmail.com

Received March 19, 2013; Accepted April 20, 2013; Published April 22, 2013

Citation: Nayak SR, Anindita M, Soren DK, Nagendra SB (2013) Spontaneous Pnemoperitoneum after Blunt Trauma Chest -Diagnostic Dilemma-Role of Diagnostic Laparoscopy - Case Report. J Trauma Treat 2: 164. doi:10.4172/21671222.1000164

Copyright: (C) 2013 Nayak SR, et al. This is an open-access article distributed under the terms of the Creative Commons Attribution License, which permits unrestricted use, distribution, and reproduction in any medium, provided the original author and source are credited. 
obtained, the sub diaphragmatic air and pneumothorax disappeared, chest tube removed and the patient discharged home on the seventh postoperative day.

\section{Case Discussion}

Pneumoperitoneum in trauma patients reliably indicates gastrointestinal perforation in $90 \%$ of cases and usually requires exploratory laparotomy [1]. The remaining $10 \%$ of cases are attributable to a variety of nonpathologic causes that result in free sub diaphragmatic air but may not require surgical intervention. Such cases have been referred to as "idiopathic" or "spontaneous" PP. The origin of air in these cases generally can be attributed some of the benign causes.The causes are air leakage from pneumatosis cystoides intestinalis, a small perforated duodenal ulcer, a leak from a colonic diverticulum, insufflations of air through the female genital tract, chronic obstructive pulmonary disease, cardiopulmonary resuscitation, or mechanical ventilation $[1,2]$ Spontaneous Pneumoperitoneum(SP) has been attributed to several thoracic causes, such as traumas (including barotraumas), pneumothorax and bronchoperitoneal fistulas [2]. Air can reach the peritoneal cavity in both blunt and penetrating chest traumas, following normal or abnormal pathways, i.e., diaphragmatic interruptions in the former case and congenital defects or post-traumatic diaphragmatic injuries in the latter case $[3,4]$. The blunt trauma patient described in the case report presented with respiratory distress with pain and distension of abdomen. Clinically and radiologicaly there was surgical emphysema and fracture ribs on left side with pneumothorax.ICTD promptly relieves the respiratory symptoms. Patterned abrasions of tyre marks (London's sign), obliterated liver dullness, tenderness on palpation of abdomen with radiological evidence of pneumothorax, and Pneumoperitonum made us dilemma for conservative / no conservative approach. Complications from missed intra-abdominal injuries can be disastrous. It has been proposed that in polytrauma cases with combined pneumothorax and pneumoperitoneum, a clinical/subclinical visceral perforation may have occurred, permitting only the leakage of air and not of bowel contents [5]. Fear of missed abdominal injury made to do diagnostic laparoscope for the present case. The laparoscopy with thorough exploration of hollow viscera and diaphragm did not show any injury or perforation. The possible explanation of his PP is that very high intrathoracic pressure following the initial impact caused pneumothorax and pneumomediastinum, leading to dissection of air through the mediastinum into the retro peritoneum and, finally to the peritoneal cavity [5]. The imprint abrasion and minimal fluid mislead us. The patho physiology of X-ray and CT-detected PP with blunt chest without bowel perforation has been variously studied. The intraabdominal pressure exceeds intrathoracic pressure by an average of approximately 20 to $30 \mathrm{~cm} \mathrm{H}_{2} \mathrm{O}$ during both inspiration and expiration, hence simple pneumothorax should not lead to PP. Even patients with tension pneumothorax develop this complication infrequently due to the rapidity of treatment or inadequate buildup of intrathoracic pressure. These findings suggest that very high intrathoracic pressure is required to cause dissection of air through the retroperitoneal space [6]. Traumatic Pneumomediastinum and then pneumoperitoneum occurs in up to $10 \%$ of cases of blunt chest trauma. In more than $95 \%$ of cases, it results from air leaking from ruptured alveoli collects in the interstitial space. As intrathoracic pressure increases, the air dissects along the sheath of adjacent vessels into the mediastinum. The air can then dissect into various spaces, including the pleural space and along the thoracic great vessels and esophagus into the retroperitoneum, where it may rupture into the peritoneal cavity and cause PP This pathophysiologic process was first described by Macklin in 1939 and now called as Macklin effect [7]. Asanza -Llorent etal reported study of two cases of pneumoperitoneum following blunt chest and abdominal trauma. In both patients laparotomy did not show bowel perforation and conservative treatment could have been provided [8]. There is a dilemma for surgeons to intervene the abdomen in X-ray and CTdetected PP in traumatic patients with concurrent pneumothorax. The findings of free fluid in peritoneal cavity, mesenteric or bowel wall thickening mandate surgical emergency. If ultrasound abdomen, CT abdomen findings are questionable and surgeon wants a conservative treatment, then serial examinations of the abdomen, frequent laboratory examinations, and constant monitoring of vital signs must be undertaken. Diagnostic laparocope may be an alternative in high index of suspicion. Masayoshi etal suggested the criteria for non surgical approach in a setting of pneumoperitoneum are (a)thorough physical examination (b)no peritoneal signs (c)pneumothorax (d)negative DPL (e) no intraperitoneal effusions in USG /CT (f )closed observation and repeated examination (g)absence of major brain injury, or altered sensorium [9]. In our case, the presence of imprint abrasions over the left half of abdomen, diffuse tenderness and minimal fluid in the abdominal cavity made us to do diagnostic laparoscopy. The use of laparoscopy for both diagnostic as well as therapeutic interventions has continued to expand. And this modality provides a viable alternative for the diagnosis of occult intra-abdominal injury following polytrauma .In the patient with combined pneumoperitoneum and pneumothorax the diagnostic laparoscopy with defined management algorithms has decreased the rate of negative and/or nontherapeutic laparotomy. This is particularly important in those patients where the potential for peritoneal violation exists without other clear indications for laparotomy [10].

\section{Conclusion}

Complications from missed intra-abdominal injuries can be disastrous. Diagnostic laparoscopy is a useful tool to assess the integrity of the peritoneum and to avoid a non-therapeutic laparotomy in stable patients.Poly trauma patient with patterned abrasions over abdomen, pnemoperitoneum and controversial answer from radiology may undergo diagnostic laparoscopy to exclude occult diaphragmatic injury or hollow viscous perforation. The diagnostic laparoscope may be the safe approach to avoid the confusion.

\section{References}

1. Sabsovich I, Desai R, Alba R, Jose Yunen and David Sammett (2008) Idiopathic pnemoperitoneum after blunt chest trauma. Surgical Rounds 31: 4.

2. Mularski RA, Ciccolo ML and Rappaport WD (1999) Nonsurgical causes ofpneumoperitoneum. .West J Med. 170: 41- 46

3. Catalano O (1995) Pneumoperitoneum caused by thoracic injury. Radiol med 89: $72-75$.

4. Kane NM, Francis IR, Burney RE, Wheatley MJ, Ellis JH, et al. (1991) Traumatic pneumoperitoneum: implications of computed tomography diagnosis. Invest Radiol 26: 574-578.

5. Gantt Cb Jr, Daniel WW, Hallenbeck GA (1977) Nonsurgical Pneumoperitoneum Am j surg 134: 411-414

6. Glauser FL, Bartlett RH (1974) Pneumoperitoneum in association with pneumothorax.Chest 66: 536-540

7. Assenza M, Passafiume F, Valesini L, Centonze L, Romeo V, et al. (2012) Pneumomediastinum and Pneumoperitoneum after Blunt Chest Trauma: The Macklin Effect. J Trauma 1.

8. Asanza-Llorente JA, Quesada-Peinado MC, Díaz-Oller J, Moreno-Montesinos 
Citation: Nayak SR, Anindita M, Soren DK, Nagendra SB (2013) Spontaneous Pnemoperitoneum after Blunt Trauma Chest -Diagnostic DilemmaRole of Diagnostic Laparoscopy - Case Report. J Trauma Treat 2: 164. doi:10.4172/2167-1222.1000164

Page 3 of 3

JM and Medina-Domínguez MT (2007) Pneumoperitoneum in polytraumatized patients without perforated hollow viscera. Cir Esp 82: 364-366.

9. Nishina M, Fujii C, Ogino R, Kobayashi R, Kohama A (2000) Pneumoperitoneum and pneumoretroperitoneum in blunt trauma patients. J Trauma 49: 565-566
10. Amin PB, Mangoti LJ, Fabin TC, Croce MA (2011) The role of laparoscopy in abdominal trauma. TRAUMA 13: 137-143 